\title{
Drift analysis and stabilization of a Fiber Bragg Grating interrogation device
}

\author{
Marvin Friedemann ${ }^{1 *}$, Sebastian Voigt ${ }^{1,2}$, Marie-Luise Werner ${ }^{1}$, Raoul Hecker ${ }^{2}$ and Jan Mehner ${ }^{1}$ \\ ${ }^{1}$ Department of Electrical Engineering and Information Technology \\ Chemnitz University of Technology \\ Chemnitz, Germany \\ ${ }^{2}$ Fibragg Diagnostics GmbH \\ Frankfurt/Main, Germany \\ *contact author: marvin.friedemann@etit.tu-chemnitz.de
}

\begin{abstract}
This paper analyzes wavelength drifts of a Fiber Bragg Grating interrogation device due to variations in different environmental conditions. First, the thermal induced wavelength shifts of the Fiber Bragg Gratings were abstracted. Then, it was possible to investigate the correlations between wavelength drifts and A) temperature of the interrogation device, B) air pressure, and $C$ ) static inclination of the interrogation device. Out of these three factors, unsteady temperature of the interrogation device proved to be the main reason for wavelength drift in our setup. Variations in air pressure were the second most important factor, whereas the static inclination of the interrogation device showed the least but still considerable effect on wavelength drifts. A temperature stabilization of the device housing temperature is introduced and a software-based offsetting of the air pressure is discussed.
\end{abstract}

Keywords - fiber-optic sensors; Fiber Bragg Grating; interrogation device; spectrometer; temperature; air pressure; inclination; drift compensation; drift prevention; long-term stability

\section{INTRODUCTION}

Optical fibers find applications in communication technology as well as in sensor systems. Fiber-optic sensors, such as Fiber-optic Fabry-Pérot Interferometer (FFPI) or Fiber Bragg Grating (FBG), can measure temperature, pressure, strain, and other parameters by non-electric detection principles. The variety of advantages, such as electromagnetic compatibility, possible multiplexing, signal linearity, and their small size make fiber-optic sensors attractive for different fields of applications [1]. Hence, optical fibers with several FBG can be utilized in catheters for medical diagnosis, for example to measure pressure profiles in the human intestine [2]. Other applications in this field could be the diagnosis of a spinal stenosis or invasive blood circulation recordings. Medical applications require a high resolution and exact measurement within a small range of pressure changes. Hence, data generated should not be subject to any other disturbance variables except pressure. Therefore, long-term stability and a reliable and reproducible outcome is necessary. The complete measurement system, consisting of the interrogation device and the optical fiber with FBGs and / or FFPI, is subject to variations of environmental conditions. Influences to the bare fiber can be compensated by reference measurement and mathematical compensation. The interrogation system itself causes drifts of the results due to interferences with changing device temperature, air pressure and device position (static inclination). Other groups are also working on stabilization methods, for example Ocean Optics Inc. with their USB Temperature Controller [3]. This article shows a compensation strategy by stabilizing the system's device temperature and by mathematical compensation for air pressure and discusses effects of static inclination of the system.

\section{THEORY}

\section{A. Properties of FBGs and FFPIs}

Optical fibers consist of a light guiding core, a cladding, and an additional coat for protection purposes. An FBG is a periodic change within the respective refractive index of the optical fiber core from a mean value $n_{\text {eff }}$ above the length $L$ with the grating periodicity $\Lambda$. Light wave interferences between these grating planes result in reflections of a wavelength peak, the Bragg wavelength $\lambda_{B}$, as shown in (1). The residual spectrum passes these discontinuities unaffected. The integration of multiple FBGs with different $\Lambda$ in only one single fiber is called wavelength multiplexing [4].

$$
\lambda_{\mathrm{B}}=2 \cdot \mathrm{n}_{\mathrm{eff}} \cdot \Lambda
$$

An FFPI is a Fabry-Pérot interferometer between the fiber tip and a reflective surface causing an interference pattern. Interference occurs by the optical path difference (OPD) due to the distance, called cavity, between fiber tip and its reflective surface [5].

\section{B. Sensing principles}

Longitudinal expansion and thermal exposure of the FBG result in a change of the Bragg wavelength. A rising ambient temperature implicates an increase of $\mathrm{n}_{\text {eff }}$, induced by the thermo-optic effect and thermal expansion. Strain, $\varepsilon$, raises the distance of grating planes $\Lambda$ and also increases $n_{\text {eff }}$ due to the photoelastic effect. Equation (2) shows the relative wavelength shift $\Delta \lambda$ subjected to strain depending on the material with its specific photo-elastic coefficient $p_{e}[4,6]$. Equation (3) states the dependency on temperature variation $\Delta \mathrm{T}$ with resulting $\Delta \lambda$ under the thermal fiber material coefficient $\mathrm{k}_{\mathrm{fT}}$. For the applied fibers $\mathrm{p}_{\mathrm{e}}$ is about 0.23 and $\mathrm{k}_{\mathrm{fT}}$ is $6.67 \cdot 10^{-6} \cdot \Delta \mathrm{T} / \mathrm{K}$ [7]. By covering the optical fiber with a polymer, it is 
possible to convert pressure to strain due to higher transversal contraction (Poisson's ratio) respectively to the compressibility of the covering polymer [8].

$$
\Delta \lambda=\left(1-p_{\mathrm{e}}\right) \cdot \lambda_{\mathrm{B}} \cdot \varepsilon
$$

$$
\Delta \lambda=\mathrm{k}_{\mathrm{fT}} \cdot \lambda_{\mathrm{B}}
$$

External impact to the FFPI, especially pressure, influences the cavity. Any variation in this distance results in changes to the OPD, whereas changes in the interference pattern represent the phase shift as well as the pressure variations [5].

\section{Interrogation and signal processing}

We used an Ibsen I-MON 512 E-USB 2.0 spectrometer, which combines transmission gratings, a polychromator, and a charge coupled device (CCD) linear image sensor. Broadband light from a superluminescent diode (SLED) is coupled into the optical fiber. The returning signal is transmitted to the spectrometer via a circulator. The signal processing principle is displayed in Fig. 1. The spectrometer has a resolution down to $0.5 \mathrm{pm}$ if the reflection peaks are ideally formed.

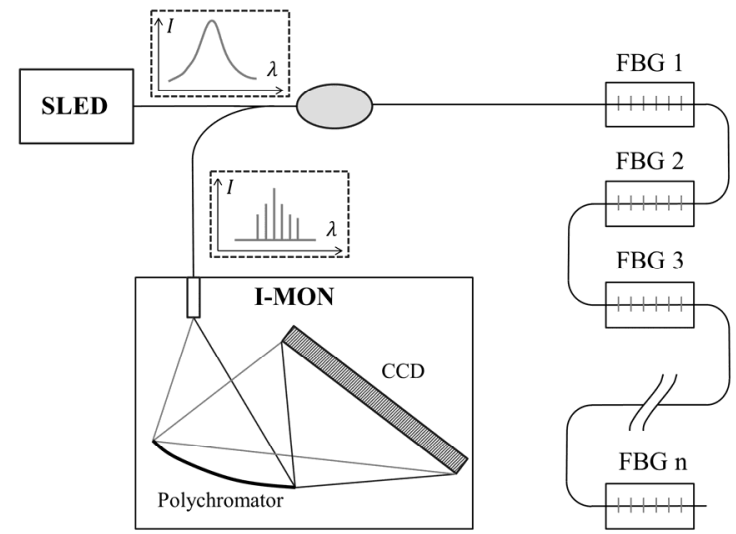

Figure 1. Interrogation principle with the I-MON combined with the external light source

The FBG used in our experiment allowed for a resolution of almost $1 \mathrm{pm}$. Using built-in sensors, device temperature, air pressure, and static inclination of the interrogation device were continuously tracked. Our interrogation device housing contained the spectrometer, the SLED light source, a printed circuit board (PCB) including an USB hub, temperature sensors (digital Tsic 306), a barometric pressure sensor (analog KP236N6165), an accelerometer (digital SCA1000-D01), a microcontroller, a display and a user interface. The recording of the sensor data as well as the wavelength spectrum tracking was done via LabView. Fig. 2 shows the main components. According to (3), ambient temperature interference on the FBG can be compensated by subtraction of the relative change in wavelength in comparison to the start of the respective measurement. The ambient temperature is recorded with a Pt1000 temperature sensor with a resolution of $0.1 \mathrm{~K}$. This compensation algorithm is implemented in our evaluation software.

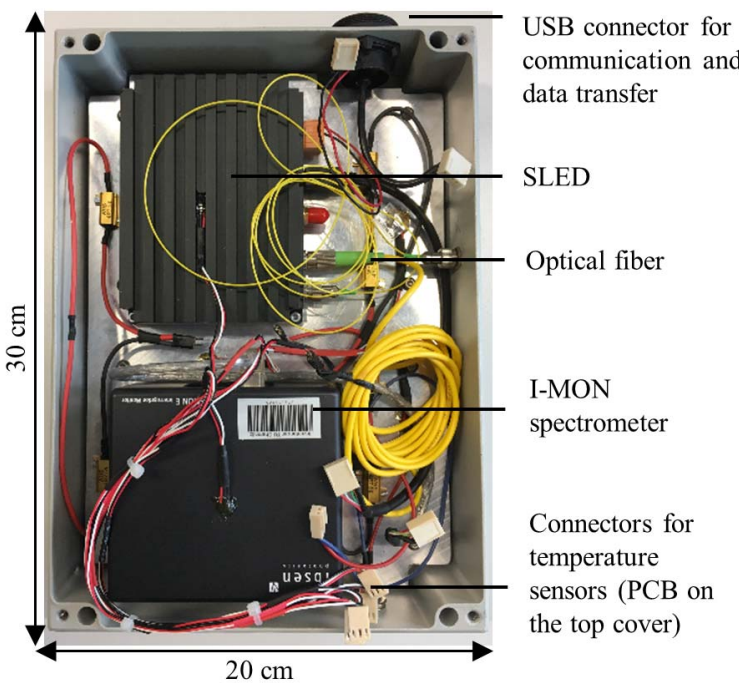

Figure 2. Interior of the interrogation device housing

\section{VARIATION OF ENVIRONMENTAL CONDITIONS}

Using an unstrained FBG and by compensating for ambient temperature, the interrogation device should show a stable wavelength readout. Nevertheless, there is a drift which is caused by fluctuations of the interrogation device temperature, air pressure, and static inclination of the interrogation device.

\section{A. Device temperature}

At a long-term wavelength measurement after power up, the temperature inside the housing of the interrogation device rises slowly and aligns close to steady state. As a consequence, a change of the Bragg wavelength is detected although it is not originating from the FBG. Fig 3. shows this wavelength drift during the period of power-up. The ambient temperature surrounding the fiber is compensated and almost constant. The device temperature rises from $23{ }^{\circ} \mathrm{C}$ to approximately $27.5^{\circ} \mathrm{C}$. Both temperature progressions are also plotted in Fig. 3. High frequency fluctuations in the wavelength plots are due to the spectrometer resolution limit of about $1 \mathrm{pm}$.

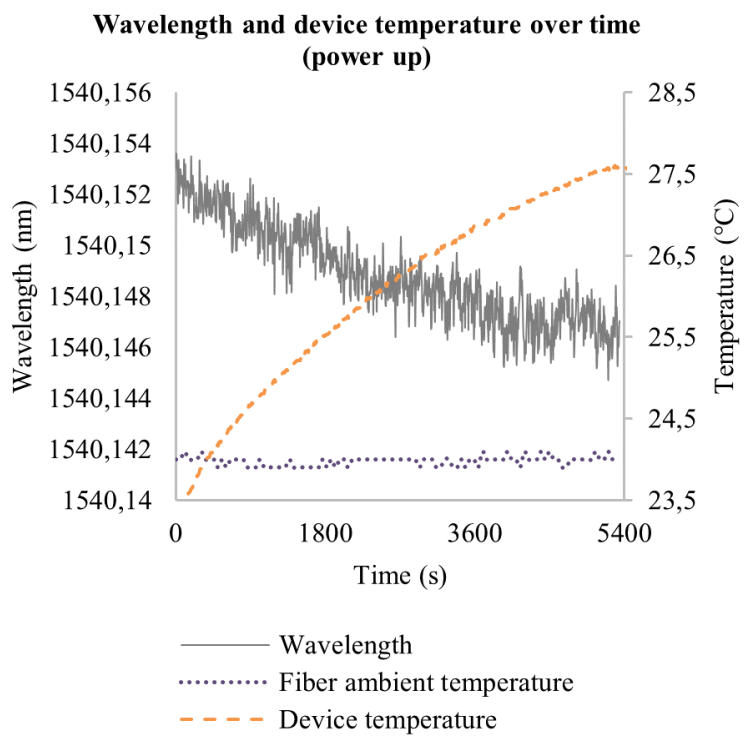

Figure 3. Wavelength drift while power up of the interrogation device with rising device temperature 
In order to exaggerate this effect, the interrogation device was exposed within a heated cabinet to temperature ramps from $27.5^{\circ} \mathrm{C}$ to $41^{\circ} \mathrm{C}$. This was repeated twice to confirm the result and to quantify the wavelength drift. At the same time, the fiber showed only minimal room temperature fluctuations which were recorded for subsequent compensation purposes.

Fig. 4 shows the drifting wavelengths readout of an unstrained FBG with a Bragg wavelength of $1540.1 \mathrm{~nm}$ with respect to the average temperature, measured by seven, evenly distributed, sensors inside the housing. For the purpose of this plot, only single wavelength values are included into the calculations, which were always recorded at the same ambient temperature in order to minimize the ambient temperature's impact, respectively the inaccuracy in ambient temperature measurements. The different offset between measurement 1 to measurements 2 and 3 is due to different absolute ambient temperatures. Relative fluctuations in this temperature surrounding the fiber could be compensated before quantifying for drift. Different absolute ambient temperature values at the beginning of the measurements cause a difference in the absolute wavelength values. This is without impact, as only relative wavelength shifts $\Delta \lambda$ correspond to the sensing principle explained in (2) and (3). The sensitivity of the interrogation device to its own temperature equals the plot's slope. An increasing device temperature $T_{d}$ causes an almost inversely proportional decrease of the wavelength readout. Normalizing the slopes with the Bragg wavelength and averaging gives a drift coefficient $\mathrm{k}_{\mathrm{dT}}$ of the interrogation device which turned out to be about $-1.22 \cdot 10^{-6} \Delta \mathrm{T}_{\mathrm{d}} / \mathrm{K}$. We assume this dependency to be linear because of its relatively narrow temperature range and its high coefficient of determination of about $98 \%$ per measurement. The obtained coefficient $\mathrm{k}_{\mathrm{dT}}$ shows that the sensitivity of the interrogation device to temperature change is at least $20 \%$ of the FBG's temperature sensitivity. $1 \mathrm{~K}$ relative increase in device temperature thus results in approximately $2 \mathrm{pm}$ wavelength reduction.

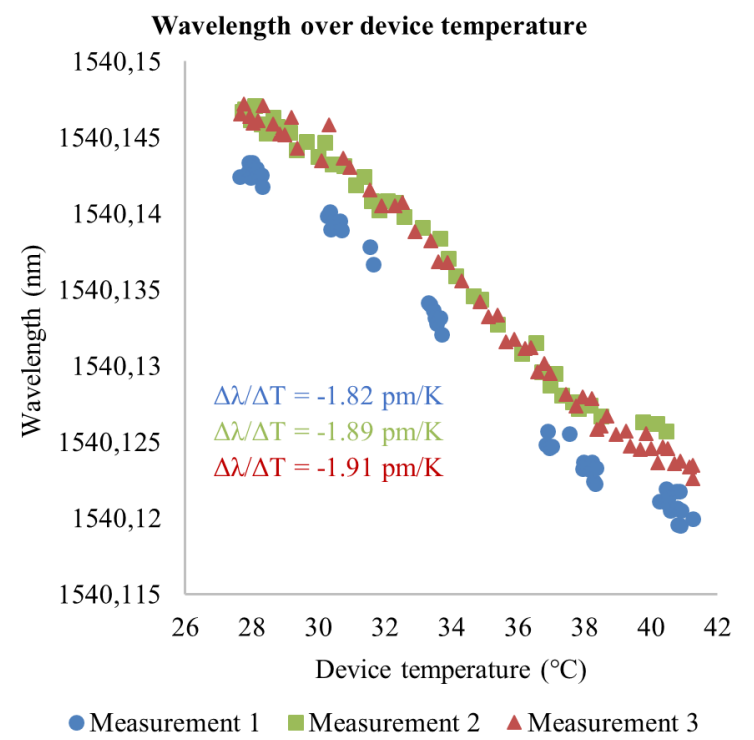

Figure 4. Recorded wavelengths when heating the interrogation device with a constant FBG as sample
The refractive index of air is temperature dependent. This physical effect is significant for light waves passing optical interfaces such as lenses [9]. Mechanical deviation inside the spectrometer is the second reason for this kind of wavelength drift. The Coefficient of Thermal Expansion (CTE) of Aluminium as housing material of the spectrometer is $23.8 \cdot 10^{-6} 1 / \mathrm{K}$ [10]. According to this CTE, a $1 \mathrm{~K}$ temperature increase raises optical distances by $>0.002 \%$ strain. Consequently, the temperature variation changes optomechanics. The CCD linear image sensor consists of 512 Pixels on a sensitive length of $12.8 \mathrm{~mm}$, on which the peaks get focused by the optomechanics. Practically, for the FBG with the Bragg wavelength of $1540.1 \mathrm{~nm}$ device temperature increase of $1 \mathrm{~K}$ causes an approximately $0.2 \mu \mathrm{m}$ light beam displacement across the length of the CCD surface.

\section{B. Air pressure}

The air pressure varies considerably due to geographical and meteorological factors as well as air movements. The barometric formula shows a logarithmic decrease of air pressure for increasing altitude. Air pressure can vary up to $3 \mathrm{hPa}$ within three hours due to meteorological dynamics. The average fluctuations of the air pressure by meteorological cycles are up to $10 \mathrm{hPa}$ per day in winter periods in temperate climate zones. Maximum values for these fluctuations are $50 \mathrm{hPa}$ per day [11]. Therefore, we investigated the interference of air pressure to the interrogation device. A pressure chamber allowed for simulating the effect of hydrostatic pressure changes. We used an optical fiber without polymer sheath which shows almost no sensitivity to hydrostatic pressure [12]. The interrogation device was exposed to a pressure ramp from approximately $800 \mathrm{hPa}$ to $1070 \mathrm{hPa}$.

Fig. 5 shows the resulting wavelength drift of the interrogation device due to hydrostatic pressure during a readout of an unstrained FBG with a Bragg wavelength of $1540.1 \mathrm{~nm}$. The different offset between measurement 2 to measurements 1 and 3 is caused by a change in ambient temperature. Relative fluctuations in this fiber temperature were compensated before drift quantification and plotting. Manual control of the pressure chamber valves is the reason for the higher deviations at the beginning and the end of the measurements. Single data points result from different sample rates between wavelength $(20 \mathrm{~Hz})$ and pressure measurement (approximately $0.14 \mathrm{~Hz}$ due to the comparatively low sample rate of the housing parameter measurements). Normalizing the slopes with the Bragg wavelength and averaging was used to find a pressure sensitivity coefficient $\mathrm{k}_{\mathrm{p}}$. With a resulting $\mathrm{k}_{\mathrm{p}}$ of about $-2.61 \cdot 10^{-7} \Delta \mathrm{p} / \mathrm{hPa}$, an air pressure rise of $10 \mathrm{hPa}$ would reduce the measured wavelengths in our FBG by approximately $4 \mathrm{pm}$. This value is a multiple of the spectrometer resolution. 


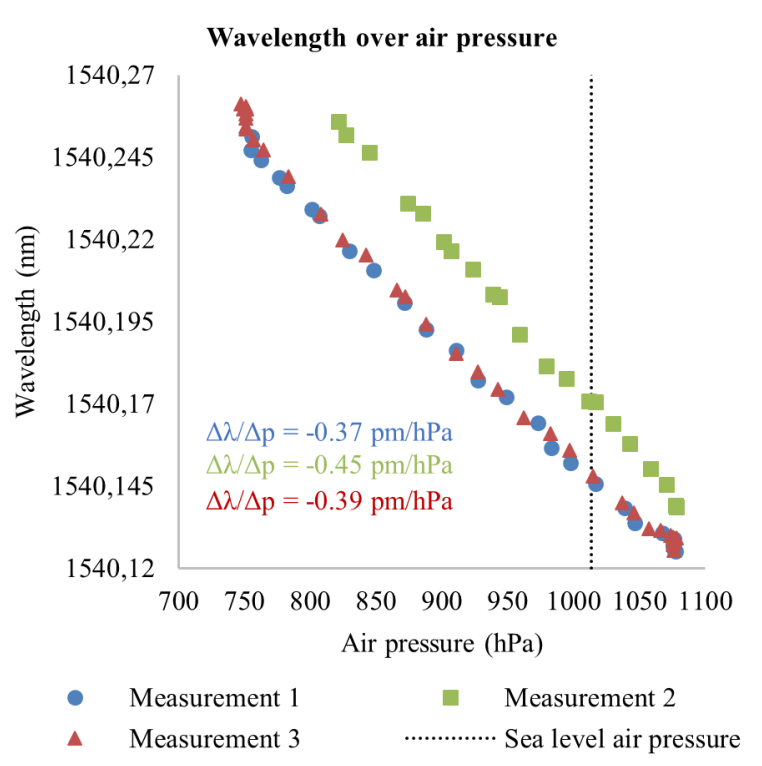

Figure 5. Recorded wavelengths when rising air pressure around the interrogation device with a constant FBG as sample

\section{Static inclination of the interrogation device}

The interrogation device housing is rectangularshaped, therefore it is possible to place the device in different positions with rectangular inclination angles. Different measurements have shown a wavelength shift when changing the position of the interrogation device with static inclination along the transverse and the longitudinal axis. Unfortunately, the different positions showed no reproducible data. The highest detectable wavelength drift at a stretch-free and temperature compensated FBG with Bragg wavelength of $1540.1 \mathrm{~nm}$ was more than $3 \mathrm{pm}$ (minimum rotation of $90^{\circ}$ around longitudinal axis). A different FBG with a Bragg wavelength of $1545.9 \mathrm{~nm}$ showed an even higher wavelength drift of almost $6 \mathrm{pm}$ (rotation of at least $90^{\circ}$ around transverse axis).

Changing the position of the interrogation device by rotation varies the direction of the underlying gravitation onto the optical components inside the spectrometer. Minimal flexibilities of the optical components (transmission gratings and concave mirror) could change their respective distances. The wavelength drifts possibly result from variations in the optical path lengths due to the technical method of wavelength discretization.

\section{COMPENSATION STRATEGIES}

With increasing precision in wavelength interrogation the significance of the drift behavior becomes higher. Without drift compensation, respectively prevention, the possible accuracy of the spectrometer is significantly reduced. Compensation and prevention strategies for the investigated, environmentally caused, wavelength drift enable long-term stability in wavelength interrogation.

\section{A. Device temperature compensation}

High precision measurements require a wavelength drift compensation. The temperature drift has an important impact on the measured wavelength due to frequently appearing fluctuations in ambient temperatures, e.g. room climate, device temperature or thermal radiation inside the application environment. Different operation modes of the interrogation device's components induce a temperature fluctuation of the device as well. A controlled heating of the device housing keeps the temperature constant independent of a range of ambient conditions. A proportional-integral (PI) control using heating resistors mounted at the base plate adjusts the temperature with an accuracy of $\pm 0.3 \mathrm{~K}$. The PI control is implemented into the microcontroller. This allows for stand alone operation of this controlled heating. For a constant device temperature, it is necessary to set this value considerably higher than the environmental temperature which can be expected during measurements. According to the I-MON datasheet, the maximum device temperature is $50{ }^{\circ} \mathrm{C}$. Hence, $40{ }^{\circ} \mathrm{C}$ is a decent choice for a sufficient difference to ambient temperature under normal ambient conditions. Operating the device at this controlled elevated temperature, the device temperature drift is almost eliminated, shown in Fig. 6. This plot presents the wavelength over the average device temperature.

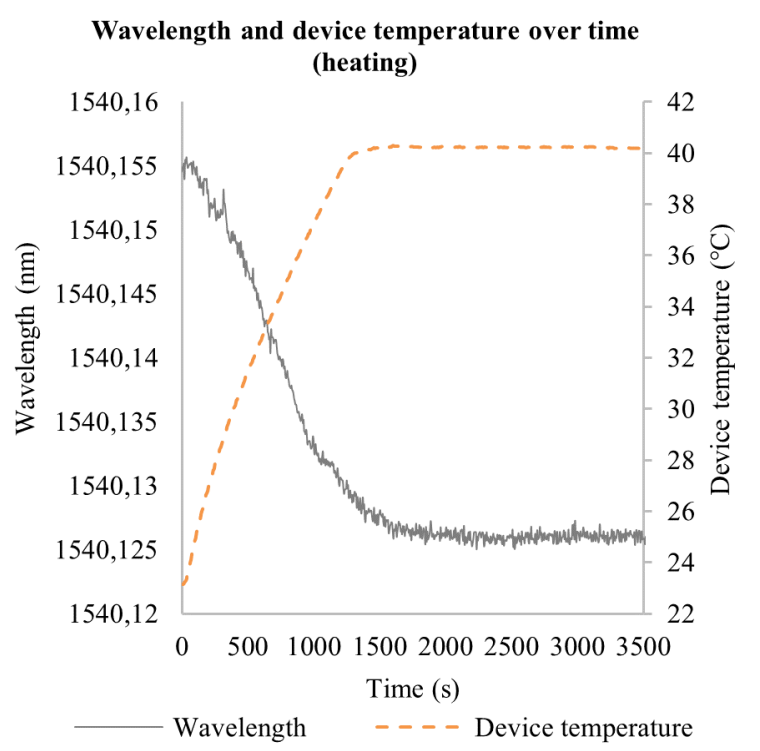

Figure 6. Wavelength plot while starting the interrogation device heating with a constant FBG as sample

\section{B. Air pressure compensation}

Assuming that the geographical position (altitude) is unchanged during the complete measuring period, a meteorological impact has still to be considered. For short measuring periods, e.g. over a few minutes, the impact of air pressure fluctuations is negligible due to their comparatively small change. Under longer term measurement conditions, the variable air pressure becomes increasingly significant [11]. We use a compensation strategy based on reference values. Therefore, it is necessary to continuously record the air pressure inside the interrogation device housing by a barometric reference pressure sensor simultaneously to wavelength measurement. The pressure compensation is similar to ambient temperature compensation: pressure indicated drifted wavelengths $\Delta \lambda$, shown in (4), are subtracted from the measured wavelengths by software. 


$$
\Delta \lambda=\mathrm{k}_{\mathrm{p}} \cdot \lambda_{\mathrm{B}}
$$

Due to the low sample rate of the housing parameter measurements this compensation strategy is not able to compensate for short term pressure changes, such as closing doors or windows.

\section{Wavelength drift prevention regarding to static inclination}

Wavelength drift prevention can be realized by an unchanged position of the interrogation device during each measurement series, so the impact of gravity on the optical components stays constant.

\section{CONCLUSION}

The impacts of variable environmental conditions and possible static inclinations on an FBG interrogation device for fiber-optic sensors were investigated experimentally. All three influence factors induce wavelength drifts, affecting long-term stability of the interrogation device. Quantification of these impacts showed the need for compensation strategies. An increasing device temperature of the interrogation device resulted in almost linear wavelength decrease with the sensitivity factor $\mathrm{k}_{\mathrm{dT}}$ of about $-1.22 \cdot 10^{-6} \Delta \mathrm{T}_{\mathrm{d}} / \mathrm{K}$ despite the absence of any elongation in the fiber. The interrogation device showed a similar behavior regarding hydrostatic pressure sensitivity. In accordance to the determined hydrostatic pressure sensitivity coefficient $\mathrm{k}_{\mathrm{p}}$ with approximately $-2.61 \cdot 10^{-7} \Delta \mathrm{p} / \mathrm{hPa}$, the wavelengths shifted in negative correlation to air pressure. Changing the position of the interrogation device resulted in wavelength drifts of approximately $6 \mathrm{pm}$ for an FBG with the Bragg wavelength of $1545.9 \mathrm{~nm}$. It can be expected that other interrogation systems with similar optical structures produce similar wavelength drift induced by fluctuation of these environmental conditions. It was shown that operating under heated, temperature-controlled conditions prevents thermal drift. For long-term measurements, the software based pressure compensation method should be applied to remove possible meteorological air pressure fluctuations. A stable position of the interrogation device during measurement eliminates wavelength drift due to a constant force direction of gravity. Application of these compensation strategies enables long-term stability and greater precision in measurement of small signals.

\section{REFERENCES}

[1] A. Othonos and K. Kalli, Fiber Bragg gratings: Fundamentals and applications in telecommunications and sensing. Boston, Mass.: Artech House, pp. 95-147, 1999.

[2] J. W. Arkwright et al., "In-vivo demonstration of a high resolution optical fiber manometry catheter for diagnosis of gastrointestinal motility disorders," (eng), Optics express, vol. 17, no. 6, pp. 4500-4508, 2009.

[3] Ocean Optics Inc., "USB-TC Instructions", Dunedin, FL, 2012.

[4] G. Rajan, Ed., Optical fiber sensors: Advanced techniques and applications. Boca Raton: Taylor \& Francis CRC Press, pp. 208-220, 2015.

[5] Y.-J. Rao, Y. Gong, and Z.-L. Ran, Fiber-Optic Fabry-Perot sensors: An introduction. Boca Raton, FL: CRC Press Taylor \& Francis Group, pp. 1-16, 2017.

[6] M. M. Weneck, R. C. S. B. Allil, B. A. Ribeiro, and F. V. B. de Nazaré, "A Guide to Fiber Bragg Grating Sensors," in Current Trends in Short- and Long-period Fiber Gratings, C. Cuadrado-Laborde, Ed.: InTech, 2013, pp. 1-25.

[7] S. Voigt, "Drucksensorkatheter auf Basis von Faser-BraggGittern," Doctoral thesis, Chemnitz University of Technology, Chemnitz, pp. 47-48, 2011.

[8] B. Budiansky, D. C. Drucker, G. S. Kino, and J. R. Rice, "Pressure sensitivity of a clad optical fiber," (eng), Applied optics, vol. 18, no. 24, pp. 4085-4088, 1979.

[9] K. P. Birch and M. J. Downs, "An Updated Edlén Equation for the Refractive Index of Air," Metrologia, vol. 30, no. 3, pp. 155-162, 1993.

[10] K. Gieck and R. Gieck, Technische Formelsammlung, 34th ed. München: Hanser, p. Z11, 2019.

[11] B. Klose and H. Klose, Meteorologie: Eine interdisziplinäre Einführung in die Physik der Atmosphäre, 2nd ed. Berlin: Springer Spektrum, pp. 107-121, 2015.

[12] M. G. Xu, L. Reekie, Y. T. Chow, and J. P. Dakin, "Optical in-fibre grating high pressure sensor," Electron. Lett., vol. 29, no. 4, pp. 398-399, 1993. 